\title{
Mitotic regulation of TFIID: inhibition of activator-dependent transcription and changes in subcellular localization
}

\author{
Neil Segil, ${ }^{1,4,6}$ Mohamed Guermah, ${ }^{2}$ Alexander Hoffmann, ${ }^{2,5}$ Robert G. Roeder, ${ }^{2}$ and \\ Nathaniel Heintz ${ }^{1,3}$ \\ ${ }^{1}$ Laboratory of Molecular Biology, ${ }^{2}$ Laboratory of Biochemistry and Molecular Biology, and ${ }^{3}$ Howard Hughes Medical \\ Institute, Rockefeller University, New York, New York 10021 USA
}

\begin{abstract}
Mitosis in higher eukaryotes is accompanied by a general inhibition of transcription. To begin to understand the mechanisms underlying this inhibition we have examined the behavior of the general transcription factor TFIID during mitosis. Immunocytochemistry and subcellular fractionation studies indicate that the majority of TFIID is displaced from the disassembling prophase nucleus to the mitotic cytoplasm around the time of nuclear envelope breakdown. However, a subpopulation of TFIID remains associated tightly with the condensed mitotic chromosomes. Metabolic labeling of mitotic cells revealed that several subunits of TFIID undergo mitosis-specific phosphorylation, but in spite of these changes, the TFIID complex remains intact. Functional analysis of purified TFID from mitotic cells shows that phosphorylated forms are unable to direct activator-dependent transcription, but that this activity is restored upon dephosphorylation. These results demonstrate that TFIID regulation by phosphorylation is likely to have an important role in mitotic inhibition of RNA polymerase II transcription. In addition, they suggest a mechanism for regulating gene expression through the selective disruption of polymerase II promoter structures during mitosis.
\end{abstract}

[Key Words: Mitosis; transcription; TFIID; TAFs; phosphorylation; chromosome localization]

Received June 21, 1996; revised version accepted August 8, 1996.

The assembly of chromatin into a regulated network of expressing and nonexpressing genes occurs in the context of the cell cycle. The pattern of gene expression that emerges is characterized both by a general stability from one cell division to the next, as well as by a capacity to establish new patterns of stable expression in a developmentally regulated way. These changes, from one stable state to another, are likely to require the temporal disruption of chromatin structure that is imposed by passage through the different stages of the cell cycle. For instance, DNA replication is invoked not only as the time of de novo chromatin assembly, but also as a time of temporary chromatin disruption, when transcription stops and newly synthesized transcription factors or other mediators of differentiative change can gain access to the DNA (for review, see Brown 1984; Villarreal 1991; Laurenson and Rine 1992; Wolffe 1994). Similarly, the dramatic changes in nuclear and chromatin structure that occur at mitosis in higher eukaryotes are accompanied by a general inhibition of transcription (Taylor

\footnotetext{
${ }^{4}$ Present address: House Ear Institute, Department of Cell and Molecular Biology, Los Angeles, California 90067 USA.

${ }^{5}$ Present address: Department of Biology, Massachusetts Institute of Technology, Cambridge, Massachusetts 12039 USA.

${ }^{6}$ Corresponding author.
}

1960; Prescott and Bender 1962; Terasima and Tolmach 1963; Davidson 1964) and may also provide an opportunity to alter chromatin structure and hence patterns of gene expression. How initiation of RNA polymerase II (pol II) transcription is inhibited as cells enter mitosis and which elements of the transcription apparatus are targets of the mitotic inhibitory pathways are the subjects of this paper.

Mitotic inhibition of transcription was first observed in the early 1960s (Taylor 1960; Prescott and Bender 1962; Terasima and Tolmach 1963; Davidson 1964). Transcriptional silencing occurs during mitotic prophase (Prescott and Bender 1962), a time when numerous other biochemical changes, including a massive increase in protein phosphorylation, occur throughout the cell (Sahasrabuddhe et al. 1984). When nuclear envelope breakdown finally occurs at the prometaphase border, much of the contents of the nucleus mixes with the mitotic cytoplasm (see Prescott 1976). During metaphase and anaphase, transcription remains silenced, and it is not until the beginning of nuclear envelope reformation in telophase that transcription resumes (Simmons et al. 1973).

Several mechanisms for controlling the onset of mitotic transcriptional inhibition have been proposed and/ or documented (for review, see Hartl et al. 1993). These 
include a direct effect of mitotic kinases on the transcription initiation machinery (Segil et al. 1991; Hartl et al. 1993), inhibition of transcript elongation and abortion of nascent transcripts (Shermoen and O'Farrell 1991; Weisenberger and Scheer 1995), and structural constraints caused by increasing chromatin condensation as cells progress through mitosis (Johnson and Holland 1965; Farber et al. 1972; Martinez-Balbas et al. 1995). These mechanisms are not mutually exclusive, and neither the arrest of RNA elongation nor the condensation of chromatin are incompatible with mechanisms of transcriptional inhibition mediated by direct effects early in mitosis. We have shown previously that Oct- 1 is hyperphosphorylated at mitosis at a site within its homeo domain that results in an inhibition of its DNA-binding activity (Segil et al. 1991). Subsequently, mitotic phosphorylation of a number of RNA pol II transcription factors has been demonstrated, including the glucocorticoid receptor (Hsu et al. 1992) and c-myc (Luscher and Eisenman 1992). In the heat shock promoter, Martinez-Balbas et al. (1995) demonstrated recently that sites normally occupied by sequence-specific transcription factors are unoccupied during mitosis, and that the corresponding factors, like Oct-1, are displaced from the condensing chromosomes. Whether these events are mediated directly by phosphorylation or by some other process is not known (Martinez-Balbas et al. 1995).

Because pol II transcription is inhibited globally during mitosis, elements of the basal pol II transcription machinery are likely to be affected directly. In vitro, pol II preinitiation complexes (PIC) assemble on TATA boxcontaining promoters in an orderly fashion with the initial step being the binding of the transcription factor TFIID. Human TFIID is an $\sim 800 \mathrm{kD}$ protein complex that contains the TATA box-binding protein (TBP) as well as $\geqslant 12$ other TBP associated factors (TAFs) (Chiang et al. 1993; Zhou et al. 1993; for review, see Burley and Roeder 1996). TFIID binding is followed by the association of TFIIA and TFIIB and subsequently by the recruitment of the other general transcription factors TFIIE, TFIIF, TFIIH, and pol II (for review, see Zawel and Reinberg 1993). TFIID is the only factor in the PIC capable of independent, sequence-specific DNA binding and it is primarily through its TBP subunit that TFIID interacts with DNA.

The efficiency of PIC assembly is regulated by the presence of transcription factors bound to sites both proximal to and remote from the site of transcription initiation. Models for how transcription factors influence PIC assembly involve specific interactions with TFIID that, through either qualitative or quantitative effects on TFIID binding, enhance recruitment of downstream factors (Horikoshi et al. 1988a,b; Workman et al. 1988; Lieberman and Berk 1994; for review, see Zawel and Reinberg 1995; Roeder 1996; Burley and Roeder 1996). Although TBP, in the absence of associated TAFs, is capable of mediating the assembly of the PIC and directing basal level transcription in vitro, regulated transcription requires the presence of TAFs (Hori and Carey 1994). Relevant to the mechanisms underlying these events, TAFs have been shown to interact directly, not only with specific activators, but also with components of the basal transcription machinery (for review, see Zawel and Reinberg 1995; Burley and Roeder 1996). Any or all of these interactions might be targets for mitotic transcriptional inhibition.

We find that a majority of TFIID is relocalized from the interphase nucleus to the cytoplasm in late mitotic prophase. We also show that during mitosis TFIID is multiply phosphorylated and in this state is unable to direct activator-dependent transcription. This deficit is reversed by dephosphorylation in vitro, indicating that phosphorylation is likely to be a key event regulating TFIID activity during mitosis. We speculate that the disruption of the basal transcription machinery during mitosis may allow a restructuring of specific promoters as transcription restarts during the transition from mitosis to interphase.

\section{Results}

TFIID redistributes from interphase nuclei to mitotic cytoplasm in late prophase

To assess the effects of mitosis on the RNA pol II transcriptional machinery we examined the subcellular distribution of TBP and the related TFIID subunits TAF $_{\mathrm{II}} 20$ and $\mathrm{TAF}_{\mathrm{II}} 15$ during the cell cycle (Fig. 1). HeLa cells at different stages of the cell cycle were fixed and incubated with antibodies against either TBP or $\mathrm{TAF}_{\mathrm{II}} 20 / 15$. The anti-TBP monoclonal antibody used in this experiment recognizes an epitope in the nonconserved amino terminus of the human TBP protein (Lobo et al. 1992). Cells were photographed in phase contrast (Fig. 1, right column) to localize the perimeter of the cell and following staining with DAPI (Fig. 1, center column) to localize the nuclear and chromosomal DNA for comparison with the immunofluorescence (Fig. 1, left column). Immunofluorescent staining of TBP in interphase cells is confined to nuclei (Fig. 1A, orange arrows). The finely speckled appearance of the interphase nucleus suggests that TBP is confined to an unknown nuclear structure represented by these nuclear speckles. In general, staining appears throughout the nucleus, although the nucleolus is relatively poorly stained. In early prophase cells (Fig. 1A, yellow arrows), as the chromosomes begin to condense, the staining of TBP in the nucleus takes on a mottled appearance that is less uniform than in interphase and appears largely chromosomal. At this early stage, probably before nuclear envelope breakdown, the cytoplasm is free of TBP staining. At later stages of prophase, when chromosome condensation has progressed further and nuclear envelope breakdown has occurred (Fig. 1B, orange arrows|, the mitotic cytoplasm is brightly stained, indicating that TBP has redistributed throughout the mitotic cytoplasm. The diffuse staining of mitotic cytoplasm persists through metaphase (Fig. 1A, white arrows) to the early stages of telophase (Fig. 1B, yellow arrows), but at later stages staining is again concentrated over the chromosomes and reforming nuclei of the 

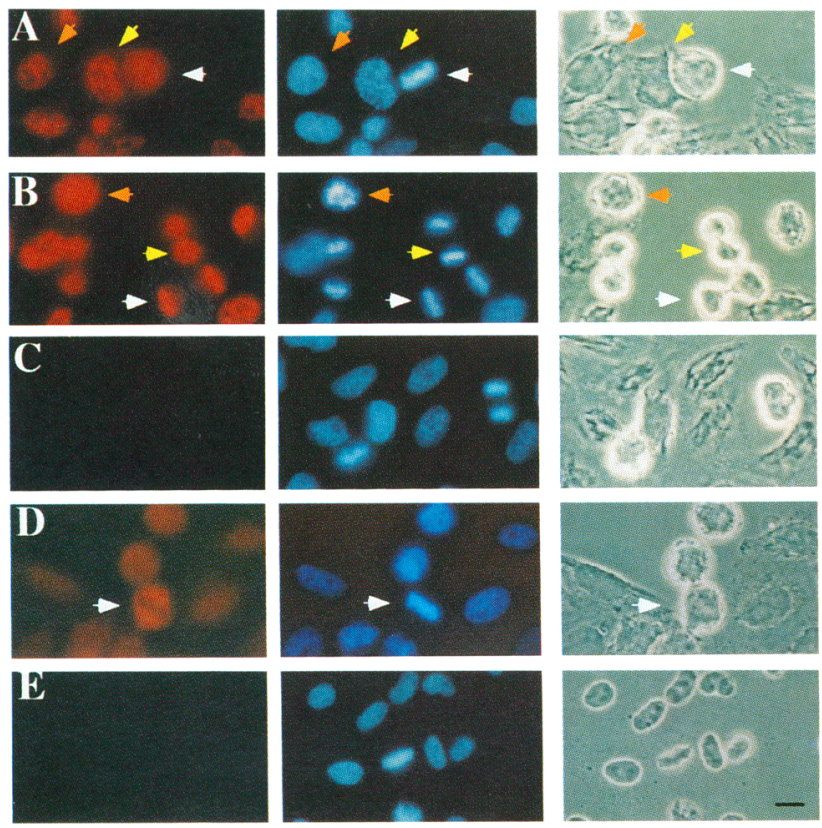

Figure 1. Subcellular distribution of TBP and $\mathrm{TAF}_{\mathrm{II}} 20 / 15$ during interphase and mitosis. HeLa cells were incubated with mouse monoclonal antibody against recombinant human TBP (N. Hernandez, Cold Spring Harbor Laboratory, Cold Spring Harbor, NY) (rows $A-C$ ) or rabbit polyclonal antibody to recombinant human $\mathrm{TAF}_{\mathrm{II}} 20 / 15$ (rows $D-E$ ) and visualized by immunofluorescent secondary antibody to mouse IgG or rabbit IgG conjugated to the fluorescent dye lissamine rhodamine (left column). The cells were counter-stained with DAPI and the same field was photographed under UV illumination to reveal the DNA staining (middle column) as well as under phase-contrast illumination (right column). (A) Orange arrows indicate an interphase cell with antibody staining confined to the nucleus; yellow arrows indicate an early prophase cell with staining still localized to chromosomal regions of the nucleus; white arrows indicate a metaphase cell with diffusely stained mitotic cytoplasm (note metaphase plate in middle column). (B) Orange arrows indicate a late prophase cell (see DNA stain) where immunofluorescence is no longer associated with condensing chromosomes and diffusely stains the mitotic cytoplasm; yellow arrows indicate one of a pair of telophase cells that have not completed cytokinesis, immunofluorescence is still located in the mitotic cytoplasm. In contrast, white arrows indicate a pair of cells at a later stage of telophase where staining has begun to concentrate over newly forming nuclei and staining in the cytoplasm has decreased. (C) No primary antibody was added as control for secondary antibody (goat anti-mouse IgG) background. (D) Cells stained with antibody to $\mathrm{TAF}_{\mathrm{II}} 20 / 15$. The white arrow indicates a cell in metaphase with diffusely staining mitotic cytoplasm and poorly stained condensed chromosomes (note condensed chromosomes lying on the metaphase plate). Other cells in field are slightly out of focus but immunofluorescent staining is always confined to interphase nuclei (data not shown). (E) No primary antibody used as control for secondary antibody (goat antirabbit $\operatorname{IgG}$ ) background. Bar, $10 \mu \mathrm{m}$.

daughter cells (Fig. 1B, white arrows). At this time there is a commensurate reduction in the staining of the cytoplasm, suggesting that the TBP is being actively relo- calized to the nucleus. Control sections showed no labeling when primary antibody was not included (Fig. 1C).

Because TBP takes part in transcription of genes transcribed by pol I and pol III as well as by pol II, we were interested in analyzing the distribution of a pol II-specific TFIID subunit. Anti-TAF ${ }_{\text {II }} 20 / 15$ staining of a metaphase cell appears in Figure 1D (white arrows). As with anti-TBP, staining is largely cytoplasmic with relatively poorly stained condensed chromosomes. Because HeLa cells "round up" when in mitosis, the interphase cells in Figure 1D are below the focal plane of the mitotic cell and so appear slightly out of focus. Nonetheless, during interphase, like anti-TBP staining, $\mathrm{TAF}_{\mathrm{II}} 20 / 15$ staining is confined to nuclei and relocalizes to the mitotic cytoplasm in late prophase; $\mathrm{TAF}_{\mathrm{II}} 20 / 15$ staining then returns to the reforming nuclei in late telophase with the same apparent time course as that for TBP (data not shown).

Although the majority of TFIID was present in the mitotic cytoplasm by this analysis, subcellular fractionation studies indicated that $10-20 \%$ of TFIID could be found in a preparation of condensed mitotic chromosomes prepared by simple NP-40 lysis and differential centrifugation (data not shown). This portion of the TFIID population that appeared to be maintained on condensed chromosomes might be overlooked in our immunofluoresence experiments because of the intensity of the staining of TBP and $\mathrm{TAF}_{\mathrm{II}} 20 / 15$ in the mitotic cytoplasm. We therefore extracted cells with nonionic detergent before fixation and TBP immunocytochemistry. A faint staining could now be seen associated with the condensed mitotic chromosomes in the absence of staining of the mitotic cytoplasm (Fig. 2A). In addition, several bright spots of TBP staining could be seen associated with mitotic chromosomes. These are likely to represent the TBP found in the pol I transcription factor SLl, which has recently been shown to remain associated with nucleolar organizing regions during mitosis (Jordan et al. 1996; Roussel et al. 1996). However, we clearly see an additional diffuse staining of the mitotic chromosomes that could represent residual TFIID that was not displaced to the mitotic cytoplasm.

To test this possibility biochemically we fractionated mitotic cells into pellet and supernatant following simple NP-40 lysis (Greenberg and Ziff 1984) and analyzed protein from equivalent numbers of cells by quantitative immunoblots. Based on this analysis, $10-20 \%$ of TBP immunoreactivity could be found associated with the chromosomal pellet, whereas a substantially smaller proportion of the $\mathrm{TAF}_{\text {II }}$ subunits were retained /data not shown). However, these chromosome preparations are relatively crude and therefore likely to be contaminated by the presence of cytoplasmic proteins. We therefore produced highly purified mitotic chromosomes using the technique of Gasser and Laemmli (1987). These were extracted and the protein analyzed by immunoblotting for the presence of general transcription factors. TBP and TAF $_{\text {II }} 20 / 15$ are clearly present in the mitotic chromosome preparation, whereas the general transcription factors TFIIA and TFIIB, as well as the transcription factor 
$\mathbf{A}$
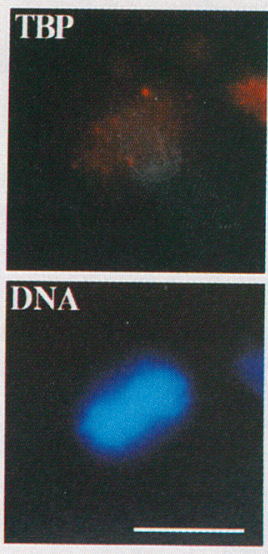

B

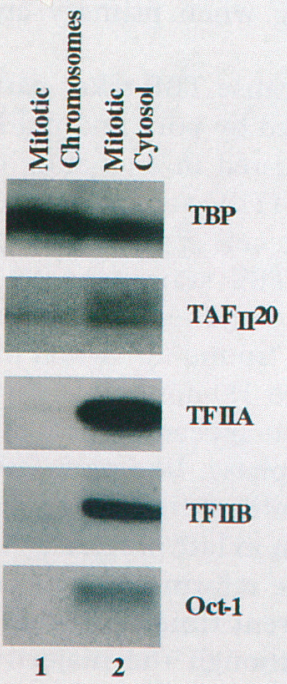

Figure 2. Analysis of subcellular localization of basal pol II transcription factors in interphase and mitosis. $(A)$ A sub-population of TBP is visible on chromosomes of mitotic cells that have been detergent extracted. HeLa cells were extracted for 2 min with $0.5 \%$ NP-40 before fixation and immunocytochemical staining for TBP with monoclonal anti-TBP (N. Hernandez, Cold Spring Harbor Laboratory, NY) (upper panel). DNA in metaphase chromosomes was stained with DAPI (lower panel). (B) Analysis of highly purified mitotic chromosomes. Chromosomes from nocodazole treated HeLa cells (mitotic index $>90 \%$ | were purified by the method of Gasser and Laemmli (1987). Protein from $\sim 2 \times 10^{6}$ chromosome clusters was compared with protein from post-chromosomal supernatant extracted from an equal number of mitotic cells by NP-40 lysis as described in Materials and Methods. Anti-TBP antibody was mouse monoclonal (from Nouria Hernandez) whereas all others were rabbit polyclonal antibodies including anti-TAF $\mathrm{TH}_{\mathrm{II}} 20 / 15$ (Hoffman et al. 1996), anti-TFIIB (Malik et al. 1993), anti-TFIIA (DeJong and Roeder 1993), and anti-Oct-1 (Roberts et al. 1991). Bar, $10 \mu \mathrm{m}$.

Oct-1, are not (Fig. 2B, lane 1). Protein from these highly purified preparations was compared with protein extracted from mitotic cytoplasm prepared by NP-40 lysis as mentioned above. This was done as a control to show that the antibodies to the general transcription factors recognize the mitotic forms (Fig. 2B, lane 2) and should not be considered quantitative. Also, although the absence of TFIIA and TFIIB in our purified chromosomal preparation is consistent with their dissociation during mitosis, this should not be taken as definitive evidence of their in vivo behavior; rather, our data indicate that under stringent conditions used for the purification of mitotic chromosomes, TFIID is retained differentially.

The dissociation of several transcription factors from mitotic chromosomes has been observed previously. Oct-1 has been shown to be preferentially extracted from mitotic cells (Segil et al. 1991), suggesting its cytoplasmic localization during mitosis, and this has been confirmed recently by immunofluorescent studies of Oct-1 localization during mitosis (Martinez-Balbas 1995; N.

Segil and N. Raju, unpubl.). At the same time there is precedence for the retention on mitotic chromosomes of TBP in the form of the pol I transcription factor SLl (Jordan et al. 1996; Roussel et al. 1996). However, the presence of TFIID on the mitotic chromosomes has not been observed previously.

\section{TFIID undergoes mitosis-specific phosphorylation}

TBP, $\mathrm{TAF}_{\mathrm{II}} 20 / 15, \mathrm{TAF}_{\mathrm{II}} 31$, and $\mathrm{TAF}_{\mathrm{II}} 80$ were immunoprecipitated independently from HeLa cells that had been metabolically labeled with ${ }^{32} \mathrm{P}$-orthophosphate and subsequently lysed in the presence of protein phosphatase and kinase inhibitors under stringent conditions that dissociate TFIID into its constituent subunits (see Materials and Methods). The immunoprecipitates were fractionated by SDS-PAGE, blotted to polyvinylidene difluoride (PVDF) membrane, and exposed for autoradiography before immunodetection of TBP and the TAFs (Fig. 3 , immunoblot). TBP from mitotic cells migrates as several bands, all of which have a slower mobility during SDS-PAGE relative to interphase TBP (Fig. 3, Immunoblot). Autoradiography reveals that all of the mitotic forms are phosphorylated (Fig. 3, Autoradiogram, open and solid arrows), indicating that several populations of differentially phosphorylated TBP exist in mitotic cells. The more slowly migrating mitotic bands (Fig. 3, TBP, solid arrow) appear here as a smear that can occasionally be resolved into a series of tightly spaced bands on immunoblots (for example, see Fig. 5, below). The relative abundance of the more slowly migrating bands appears to be less in immunoblots because they are spread out over a larger area. No comparable interphase bands exist (Fig. 3, lane 1, TBP, solid arrow). However, careful inspection of the immunoblot reveals that there are both major and minor forms of TBP in the interphase lane that run very close together (Fig. 3A, double open arrows) (observed previously by Zhou et al. 1993). All of the radiolabeled protein in the interphase lane migrates with the minor, more slowly migrating band and this band might be explained by the presence of the contaminating population of $1 \%$ to $2 \%$ of mitotic cells found in the log phase cultures used for labeling. This is consistent with its mobility, which is reduced to the same apparent extent as that of the most rapidly migrating mitotic form in which a small amount of ${ }^{32} \mathrm{P}$ is found. Because no phosphorylated $\mathrm{TAF}_{\mathrm{II}} 20 / 15$ is found contaminating the interphase sample (see Fig. 3B) it is possible that a small percentage of TBP is phosphorylated during interphase /see below).

The observation that TBP is phosphorylated during mitosis confirms the work of White et al. (1995), who noted previously the change in mobility of mitotic TBP and showed that phosphatase treatment was able to eliminate this change. To observe whether TFIID-specific TAFs are mitotically phosphorylated, we independently immunoprecipitated several TFIID-specific TAFs, $\mathrm{TAF}_{\mathrm{II}} 20 / 15$ (Fig. $3 \mathrm{~B}$ ), $\mathrm{TAF}_{\mathrm{II}} 31$ (Fig. $3 \mathrm{C}$ ), and $\mathrm{TAF}_{\mathrm{II}} 80$ (Fig. 3D). They have been shown to interact directly with TBP in vitro (Hisatake et al. 1995; Mengus et al. 1995; Hoff- 
$\mathbf{A}$
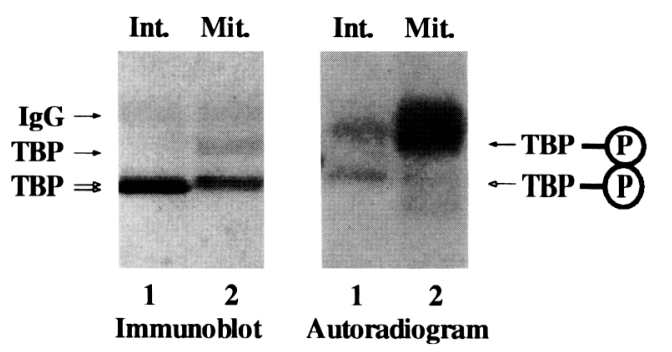

B

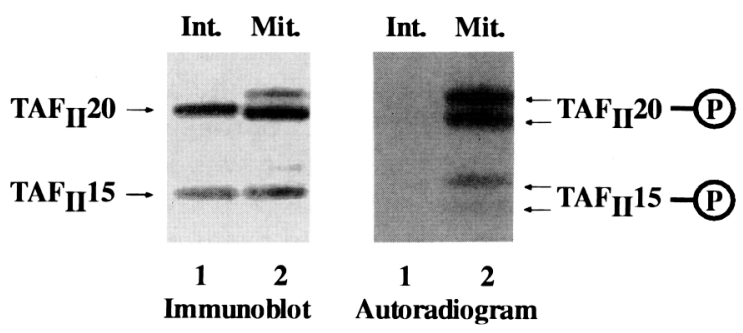

C

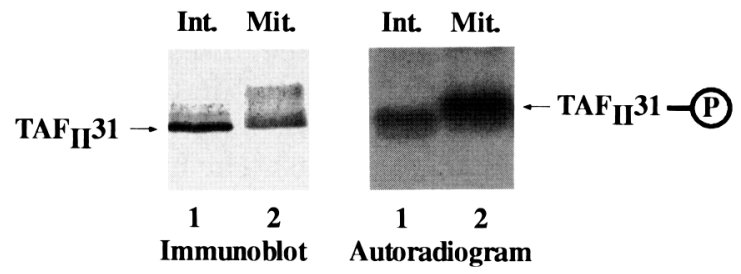

D

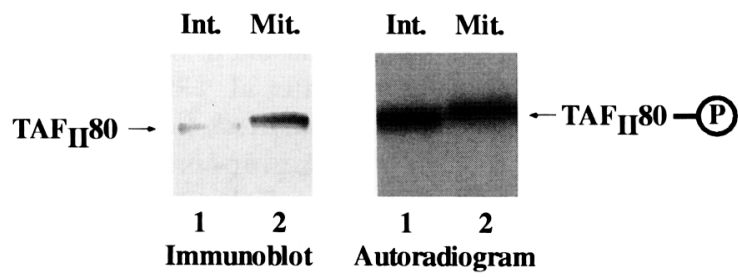

Figure 3. In vivo analysis of TBP, $\operatorname{TAF}_{11} 20 / 15, \operatorname{TAF}_{I I} 31$, and $\mathrm{TAF}_{\mathrm{II}} 80$ phosphorylation in interphase and mitotic HeLa cells. Cells, either log phase (interphase) or nocodazole-treated (mitotic) were grown in spinner culture and metabolically-labeled with ${ }^{32}$ P-labeled orthophosphate. $(A) \mathrm{TBP} ;(B) \mathrm{TAF}_{\mathrm{II}} 20 / 15 ;|C|$ $\mathrm{TAF}_{\mathrm{II}} 31 ;(D) \mathrm{TAF}_{\mathrm{II}} 80$ were immunoprecipitated independently from lysates under stringent conditions (see Materials and Methods), fractionated by SDS-PAGE, blotted to PVDF membrane, and probed for TBP and TAFs with specific antibodies: (A) Monoclonal anti-TBP (N. Thompson, McArdle Laboratories, Madison, WI); $(B)$ anti-TAF $20 / 15$ (Hoffman et al. 1996); $(C)$ anti-TAF ${ }_{\mathrm{II}} \mathrm{r} 31$ (M. Guermah, unpubl.); and $(D)$ anti-TAF $\mathrm{TA}_{\mathrm{II}} 80$ (Hisatake et al. 1995). Primary antibodies were detected by alkaline phosphatase conjugated secondary antibodies (immunoblots, left) prior to exposure for autoradiography (autoradiograms, right).

mann and Roeder 1996) and they serve here as a markers for TFIID, because unlike TBP, they are not present in transcription complexes involved in pol I and pol III transcription (Chiang et al. 1993). Immunoprecipitation of these TAFs from metabolically labeled cells shows that they too are phosphorylated specifically in mitosis (Fig. 3). Little or no ${ }^{32} \mathrm{P}$ label is associated with interphase
$\mathrm{TAF}_{\mathrm{II}} 20 / 15$ (Fig. 3B), whereas at least two forms of phosphorylated $\mathrm{TAF}_{\mathrm{II}} 20$ are present during mitosis, as are two forms of the related protein $\mathrm{TAF}_{\mathrm{II}} 15 . \mathrm{TAF}_{\mathrm{II}} 31$ and $\mathrm{TAF}_{\mathrm{II}} 80$ are phosphorylated during interphase, but undergo a hyperphosphorylation during mitosis (Fig. 3C,D) that is the most likely explanation for the change in mobility relative to that of the interphase form.

Although the mitotic phosphorylation of multiple TAFs correlates with transcriptional inhibition and mitotic relocalization of TFIID, it does not elucidate the effects of these modifications on TFIID subunit structure or the ability of TFIID to participate in transcriptional events. The results of studies addressing these questions follow.

TBP and TFIID subunits remain associated in their mitotically phosphorylated states

TFIID purified from higher eukaryotes forms a tightly associated complex (Dynlacht et al. 1991; Zhou et al. 1992; Chiang et al. 1993; Zhou et al. 1993). The correlation between the mitotic phosphorylation and subcellular relocalization of TBP and $\mathrm{TAF}_{\mathrm{II}} 20 / 15$ led us to investigate whether the TFIID complex remains intact in its mitotically phosphorylated state. Immunoprecipitation of the TFIID complex from mitotic whole-cell extracts under nondenaturing conditions (BC400) led to the coimmunoprecipitation of all TFIID subunits analyzed (Fig. 4A), indicating that TFIID remains intact in the mitotic cytoplasm. The coimmunoprecipitations of TBP, $\mathrm{TAF}_{\mathrm{II}} 20 / 15$, and $\mathrm{TAF}_{\mathrm{II}} 250$ are illustrated in Figure $4 \mathrm{~B}$ as examples. Neither TBP nor TAFs were present in immunoprecipitations using polyclonal anti-Oct- 1 as a control (Fig. 4B, lanes 5 and 6). TFIID complexes from mitotic extracts contained TBP, $\operatorname{TAF}_{\mathrm{II}} 20 / 15, \mathrm{TAF}_{\mathrm{II}} 31$, and $\mathrm{TAF}_{\mathrm{II}} 80$ in their phosphorylated forms as judged by their relative mobility on SDS-PAGE (Fig. $4 \mathrm{~B}_{\text {; }}$ data not shown). Although we have not been able to observe all species of mitotic TBP in the $\mathrm{TAF}_{\mathrm{II}} 20 / 15$ coimmunoprecipitates, the major band shown runs with slower mobility than the interphase form; as shown in Figure 3, this band is mitotically phosphorylated, albeit to a lesser extent than the more slowly migrating bands. Because TBP takes part in pol I, pol II, and pol III transcription, it is possible that there are three or more differentially phosphorylated populations of TBP that we have failed to distinguish in Figure 3. In conclusion, Figure 4 indicates that mitotic phosphorylation does not disrupt TFIID complexes that have become dispersed in the mitotic cytoplasm. Nonetheless, others have reported the isolation of TFIID complexes with differences in TAF components (Mengus et al. 1995) and it remains possible that the specific subunit composition of mitotic TFIID differs from its interphase counterpart.

Mitotically phosphorylated TFIID is unable to support activator-dependent transcription in vitro

The ability of TFIID to mediate transcriptional activation is dependent on the interaction of specific TAFs 
A

Subunits Present in Mitotic TFIID

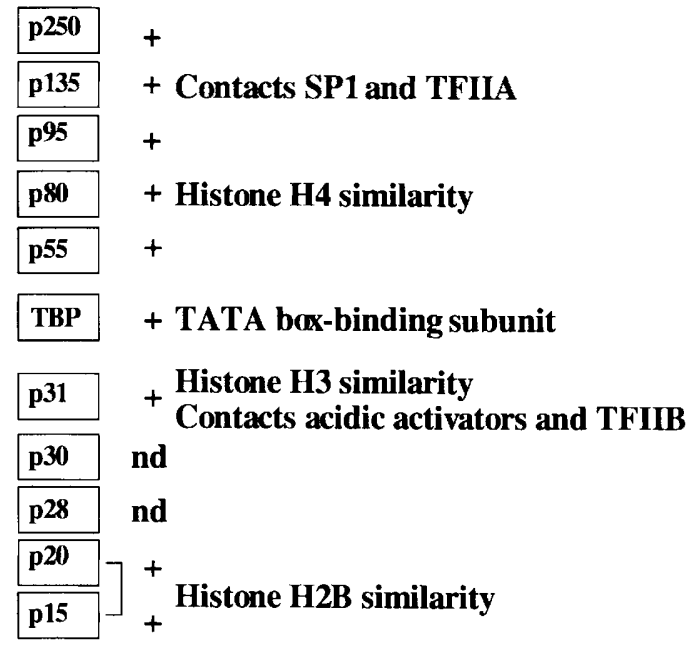

B

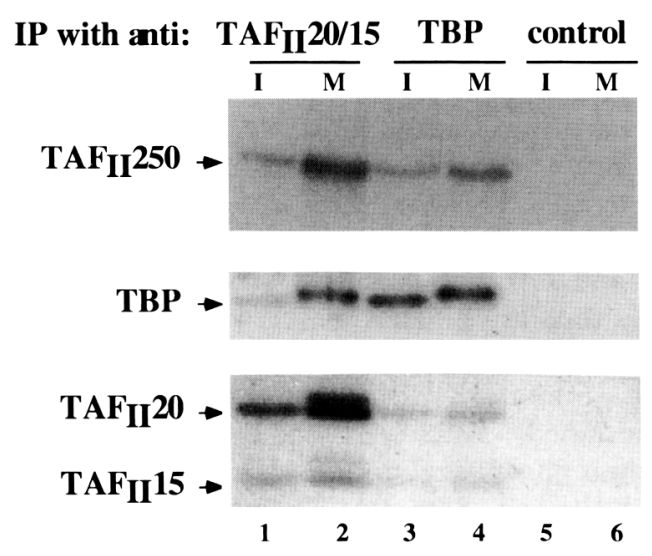

Figure 4. TFIID remains intact following mitotic phosphorylation. (A) Summary of TFIID subunits coimmnoprecipitated with antibodies to either TBP and $\mathrm{TAF}_{11} 20 / 15 .|B|$ Examples of coimmunoprecipitations summarized in $A$. Whole-cell extracts of interphase (lanes 1,3,5) and mitotic cells (lanes 2,4,6) were prepared as before (Segil et al. 1991) except that phosphatase and kinase inhibitors were included at all stages of preparation and the extracts were not dialyzed before immunoprecipitation with either anti-TBP, anti-TAF $1 \mathrm{II} 20 / 15$, or anti-Oct- 1 as control. Immunoprecipitates were fractionated by SDS-PAGE, blotted to PVDF membrane and coimmunoprecipitating proteins were detected with specific polyclonal antibodies followed by ${ }^{125} \mathrm{I}$ protein A. (nd) Not determined.

with different classes of transcriptional activators (for review, see Burley and Roeder 1996; Goodrich et al. 1996). To assess the effects of mitotic phosphorylation on the ability of TFIID to direct both basal and activatordependent transcription, we used TFIID purified from either interphase or mitotic HeLa cells expressing a
FLAG-tagged TBP molecule (f:TFIID). Purified f:TFIID was tested in a reconstituted in vitro transcription system that consists of recombinant and highly purified general transcription factors and RNA pol II from HeLa cells and that is inactive in the absence of added TBP or TFIID (Chiang et al. 1993; data not shown). The ability of mitotic TFIID to support basal as well as activator-dependent transcription was tested simultaneously using two templates whose correctly initiated products could be differentiated by the size of their transcribed G-less cassettes (Chiang et al. 1993). Basal transcription was assayed on a template containing the adenovirus major late promoter TATA box and initiator sequence, whereas activator-dependent transcription was assayed using the TATA box from the HIV-LTR and initiator sequence from the adenovirus major late promoter cloned downstream of a synthetic enhancer containing five copies of the GAL4 DNA-binding site (Chiang et al. 1993). Transcription was tested in the absence or presence of purified, bacterially expressed activator proteins consisting of the GAL4 DNA-binding protein fused to the transcriptional activation domain from the tumor suppresser protein p53 or to the glutamine-rich activation domain of SP1 (see Materials and Methods). The p53 activation domain has been shown to interact directly and specifically with $\mathrm{dTAF}_{\mathrm{II}} 42$, and $\mathrm{dTAF}_{\mathrm{II}} 62$ as well as with their human homologs $\mathrm{hTAF}_{\mathrm{tI}} 31$ and $\mathrm{hTAF}_{\mathrm{II}} 80$ (Lu and Levine 1995; Thut et al. 1995). In addition, activator-dependent transcription was tested with the glutamine-rich activation domain of SP1, which has been shown to interact directly with $\operatorname{dTAF}_{\text {II }} 110$ (Chen et al. 1994), the homolog of $\mathrm{hTAF}_{\mathrm{II}} 135$. f:TFIID was purified from either interphase or mitotic cells by immunoprecipitation with anti-FLAG epitope antibodies. Following immunoprecipitation, immobilized TFIID was washed to remove phosphatase inhibitors and then peptide eluted /Chiang et al. 1993). f:TFIID preparations were normalized by immunoblotting and phosphoimage analysis of the TBP component of the purified TFIID (Fig. 5A). Purification was carried out in the presence or absence of phosphatase inhibitors and immunoblot analysis showed that both TBP and $\mathrm{TAF}_{\mathrm{II}} 20 / 15$ are dephosphorylated by endogenous phosphatases during the purification process if prepared in the absence of phosphatase inhibitors /Fig. $5 \mathrm{~A}$; note the loss of slowly migrating bands in lane 3 relative to 4 ).

The preparations analyzed in Figure 5A were used to reconstitute both basal and activator-dependent transcription. The results show that although there was little difference $(-50 \%)$ between the basal transcription rate supported by TFIID from interphase versus mitotic cells (Fig. 5B,C, cf. lanes 3 and 7), mitotically phosphorylated f:TFIID was 9- to 10-fold less active than interphase f:TFIID in supporting activator-dependent transcription when tested with the p53 activation domain (Fig. 5B, cf. lanes 4 and 8 ). Likewise, mitotic f:TFIID was $\sim 5$-fold less active when tested with the SPl activation domain (Fig. 5C, cf. lanes 4 and 8). However, the dephosphorylated forms of mitotic f:TFIID prepared by the omission of phosphatase inhibitors were restored to interphase 
A

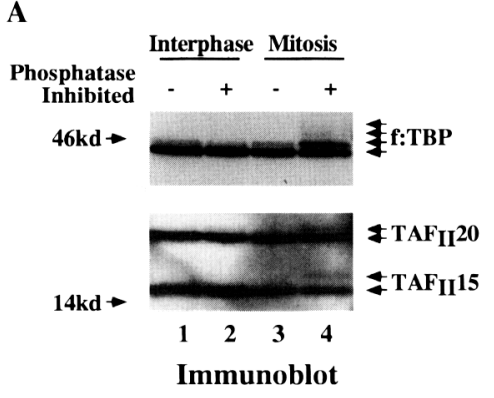

B
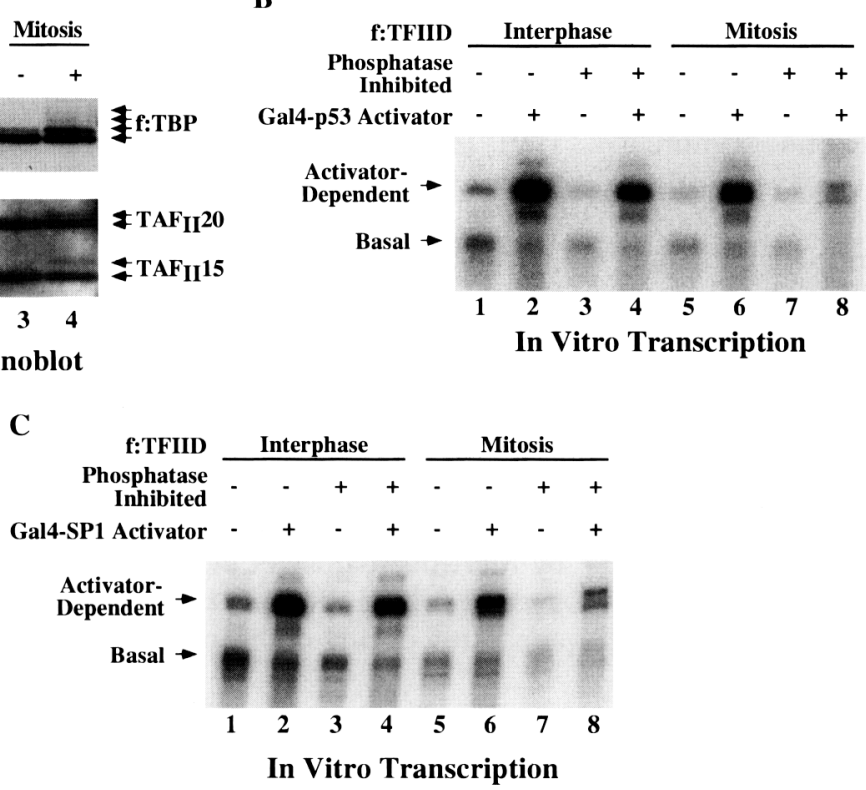

Figure 5. Mitotic inhibition of activatordependent TFIID activity. (A) Purified f:TFIID from either interphase or mitotic whole-cell extracts was prepared in either the presence or absence of phosphatase inhibitors as described in Materials and Methods. Phosphorylation state of TBP and $\mathrm{TAF}_{\mathrm{II}} 20 / 15$ was monitored by the presence or absence of bands of slower mobility as seen in lane 4 (arrows). Mitotic TBP and $\mathrm{TAF}_{\mathrm{II}} 20 / 15$ are dephosphorylated by endogenous phosphatases if samples are prepared in the absence of phosphatase inhibitors (cf. lanes 3 and 4). f:TFIID preparations were normalized for TBP content by phosphoimage analysis of ${ }^{125}$ I-protein A bound to anti-TBP. $(B, C)$ Analysis of $\mathrm{f}$ :TFIID activity by in vitro transcription in a reconstituted system consisting of highly purified and recombinant basal transcription factors (Chiang et al. 1993). Preparations shown in $A$ were tested for their ability to support either basal or activator-dependent transcription (arrows).

Activator-dependent transcription was tested by the addition of the activation domains from either the p53 $(B)$ or the SP1 $(C)$ transcription factors fused to the DNA-binding domain of the GAL4 protein. The GAL4 DNA-binding domain alone had no effect on basal transcription activity from either promoter and the system was dependent on the addition of TFIID /Chiang et al. 1993; M. Guermah and R.G. Roeder, unpubl.). Basal and activator-dependent transcription were analyzed simultaneously with plasmids containing G-less cassettes of differing lengths (Chiang et al. 1993) and with (activator-dependent) or without (basal) GAL4-binding sites in their promoters (see Materials and Methods).

levels of activity (Fig. 5B, cf. lane 6 with lanes 2 and 4). Phosphatase inhibitors were not present in the transcription reactions because they were omitted during the final washes of the f:TFIID purification before peptide elution. Three separate f:TFIID preparations were tested with the same results. These results show that TFIID activity is regulated by phosphorylation during mitosis and, to our knowledge, represent the first example of the regulation of TFIID by post-translational modification.

\section{Discussion}

\section{Mitotic TFIID phosphorylation leads to the inhibition of activator-dependent in vitro transcription}

The results presented here provide evidence that the regulation of TFIID activity by phosphorylation may be an important general component of the mitotic inhibition of pol II transcription. We have found that TBP and its associated TAFs are phosphorylated in HeLa cells during mitosis (Fig. 3), and that this event correlates with the inactivation of the normal ability of TFIID to direct activator-dependent transcription in vitro (Fig. 5). Although TBP alone can support basal transcriptional activity in vitro, activator-dependent transcription requires the presence of TAFs (for review, see Burley and Roeder 1996; Goodrich et al. 1996). Whereas the precise mechanism of transcriptional activation is not understood, the direct interaction of transcription factor activation domains with specific TAFs has been shown to be important for activation to occur (Chen et al. 1994; Lu and Levine 1995; Thut et al. 1995). Based on these observations we hypothesize that phosphorylation of TAFs directly interferes with these interactions. It will be important in the future to correlate the sites of TAF phosphorylation with the sites of TAF-activator interaction.

The inactivation of TFIID by phosphorylation is consistent with recent reports on the mitotic inhibition of pol III transcription in vitro. Hartl et al. (1993) showed that transcription ceased when Xenopus oocyte extracts are induced to enter a mitotic-like state. This event was dependent on the activity of p34-Cdc2 kinase and independent of chromatin condensation. The target of this inhibition was the basal pol III transcription factor TFIIIB (Gottesfeld et al. 1994), which, like TFIID, contains TBP as an essential subunit. The mitotic inactivation of TFIIIB was confirmed recently in a study of pol III transcription in mitotic extracts from HeLa cells (White et al. 1995). In this same study, the authors noted that TBP from mitotic cells underwent a mobility shift in SDS-PAGE that was susceptible to phosphatases, indicative of its phosphorylation. However, as in the earlier work, the phosphorylation state of the TBP component of TFIIIB did not correlate with pol III transcriptional inhibition (Gottesfeld et al. 1994; White et al. 1995), leading to the suggestion that another component of the TFIIIB complex is the target. Likewise, the TBP moiety of TFIID, although specifically phosphorylated during mitosis (Fig. 3A), may not be the relevant target for the inactivation of pol II transcription, although the small 
but consistent effect on basal transcription could be a result of this modification. The known requirement for TAFs in activator-dependent transcription, combined with our observation that multiple subunits of TFIID are phosphorylated at mitosis, suggests that one or more TAFs is the relevant target in the inhibition of activatordependent transcription.

The nature of the mitotic kinases responsible for TFIID phosphorylation are not known. Although the importance of $\mathrm{p} 34-\mathrm{Cdc} 2$ kinase in the onset of mitotic inhibition of RNA pol III transcription has been demonstrated (Hartl et al. 1993; Gottesfeld et al. 1994; White et al. 1995), a direct role for p34-Cdc2 has not. Likewise, the phosphatases responsible for dephosphorylating TFIID as the cells re-enter $G_{1}$ are unknown. The ability of mitotic whole-cell extracts to dephosphorylate TFIID during preparation and dialysis suggests either that the endogenous phosphatases responsible for the dephosphorylation of TFIID during the mitosis to $G_{1}$ transition are active during mitosis or become active as a result of extraction.

\section{The function(s) of transcriptional inhibition during mitosis}

At least two hypotheses are tenable as explanations of the effects of mitosis on the transcription apparatus. First, there may be a need to strip chromatin of some proteins in order to condense chromosomes sufficiently for segregation to occur efficiently (Nurse 1990). Second, transcription complex destabilization during mitosis may be a mechanism for re-assorting specific transcription factors and remodeling promoter structure in preparation for changes in gene expression. These two hypotheses are not incompatible and the timing of mitotic events may be crucial for understanding the role of each during mitosis. The onset of mitotic transcriptional inhibition is reported to occur during prophase (Prescott and Bender 1962) and so precedes the relocalization of TFIID that occurs at the time of nuclear envelope breakdown (Fig. 1). Although not easily visible, anti-TBP staining of the partially condensed chromosomes of early prophase cells appears to be largely chromosomal with weaker staining in the intrachromosomal space (Fig. 1B). This observation suggests that the bulk of TFIID is chromosome-associated during interphase and that it only dissociates at the time of nuclear envelope breakdown. In addition, the presence of multiple species of mitotically phosphorylated TAFs, as seen in the case of TAF $_{\text {II }} 20 / 15$ (Fig. 3), indicates that TFIID undergoes differential phosphorylation and suggests that different populations of TFIID exist. These may arise because of context-dependent phosphorylation. Accordingly, TFIID transcriptional inactivation and chromosome dissociation could be separable events mediated, as suggested above, by different phosphorylation events.

This two-step scenario is strongly supported by the observation that in the case of pol III transcription in vitro, mitotic kinases can mediate transcriptional inhibition in the absence of chromatin condensation (Hartl et al. 1993). The stripping of transcription factors from chromatin can be seen in the context of the changing patterns of post-translational modification that many transcription factors undergo during mitosis (Segil et al. 1991; Hsu et al. 1992; Luscher and Eisenman 1992; Martinez-Balbas et al. 1995). Even in those cases where no change in DNA-binding activity during mitosis can be detected (Martinez-Balbas et al. 1995), the intrinsic affinities of transcription factor complexes, including those with the TFIID complex, may be affected by phosphorylation events that compromise protein-protein interactions both at promoters and at nonspecific sites in bulk chromatin and therefore make dissociation from DNA a favored state. Alternatively, changes in the charge structure of mitotic chromosomes, as suggested by Martinez-Balbas et al. (1995), could still have an important role in the final dissociation of the transcription apparatus during chromosome condensation.

\section{Stable transcription complexes during mitosis}

We have observed that $10-20 \%$ of the TFIID population remains tightly associated with the condensed mitotic chromosomes following nuclear envelope breakdown (Fig. 2; data not shown). Precedence for the retention of components of the transcription apparatus on mitotic chromosomes can be found in the reports that RNA pol I and the pol I transcription factor upstream binding factor (UBF) are localized to the dispersed nucleolar organizing regions (NORs) during mitosis (Matsui 1979; Scheer and Rose 1984; Roussel et al. 1993). These observations have recently been extended to include the presence of SL1, the TBP-containing transcription factor required for pol I transcription (Jordan et al. 1996; Roussel et al. 1996). It seems likely that some of the phosphorylated species of TBP that we have observed are present at these sites and that it is the phosphorylation of TBP and associated pol I-specific TAFs that is responsible for pol I transcriptional inhibition.

Sequence analysis of several TAFs indicates that three of them show limited sequence homology with core histones (Kokubo et al. 1994; Hisatake et al. 1995; Hoffmann and Roeder 1996), which in the case of dTAF $_{\text {II }} 42$ and $\operatorname{dTAF}_{\mathrm{II}} 62$, translates into nearly identical structural homology within the histone core domains (Xie et al. 1996). Interactions between these TAFs appear to parallel exactly the specificity of the interactions of their histone homologs (Hoffmann et al. 1996) and this has led to the hypothesis that they form a nucleosome-like core structure within the TFIID complex (Kokubo et al. 1994; Hisatake et al. 1995; Hoffmann et al. 1996; Xie et al. 1996). If such a structure exists, it might facilitate the packing of TFIID into the condensed, higher-order structure of mitotic chromosomes. Our demonstration that like the core histones, the histone-like TAFs undergo post-translational modification during mitosis strengthens the contention that these proteins are functioning in a nucleosome-like manner in the context of TFIID.

In spite of a massive efflux of proteins from the disassembling prophase nucleus (Prescott 1976), early reports 
show that the pattern of DNase I sensitivity of the metaphase chromosomes correlates with regions that are actively transcribed during interphase (Kerem et al. 1984). This supports the notion that chromatin structure, and therefore some promoter proteins, are retained on the chromosomes during mitosis. Recent reports suggest that the persistence of transcription factor footprints during mitosis varies from promoter to promoter. For instance, in vivo footprinting of the human phosphoglycerate kinase gene promoter indicates that upstream transcription factor binding on this TATA-less promoter is lost during mitosis (Hershkovitz and Riggs 1995). The same is true in the case of the human heat shock gene promoter (Martinez-Balbas et al. 1995). However, by immunocytochemical analysis, several transcription factors are reported to be maintained on metaphase chromosomes including the Serum Response Factor (Gauthier-Rouviere et al. 1991), GAGA factor (Raff et al. 1994), and AP-2 (Martinez-Balbas et al. 1995) and an in vivo footprint located over the transcriptional initiation sequence of the dihydrofolate reductase gene is maintained during mitosis (Nicholas Heintz, pers. comm.). Our observation that the population of TFIID splits into chromosomal and nonchromosomal parts during mitosis could reflect this diversity in promoter behavior. The selective retention of specific promoter proteins or structures could be part of a system of regulation that marks genes for rapid reassembly of PICs when cells reenter the $\mathrm{G}_{1}$ phase of the cell cycle. Alternatively, the mitotic displacement of some transcription factors and the disruption of PICs could allow the re-assortment of transcription factors with similar DNA-binding properties but different activation properties.

\section{Conclusion}

The establishment of tissue-specific and developmentally regulated gene expression is thought to be a reflection of the particular combination of transcription factors expressed and bound at specific times during the differentiation process (Brown 1984; Weintraub 1985). Activation and repression of RNA pol II transcription in higher eukaryotes involves the interaction of the basal transcription machinery with these regulatory factors (Tjian and Maniatis 1994; Roeder 1996). Our observations suggest a model in which differential phosphorylation of TFIID during mitosis could contribute to the selective disassembly of some transcription complexes, whereas other complexes are maintained stably. This scenario could help to explain both the general stability of the transcriptional network from one cell division to the next, while at the same time allowing differentiative change in promoter structure to occur in a cell cycledependent manner.

\section{Materials and methods \\ Cell culture and synchronization}

HeLa cells were grown either on cover slips in Dulbecco's minimal essential medium (DMEM) with 10\% bovine calf serum for use in immunocytochemistry or in spinner culture in Joklik's medium with $5 \%$ bovine calf serum for extracts and lysates. Synchronization of spinner cultures was accomplished by treatment of $\log$ phase cultures with nocodazole $1600 \mathrm{ng} / \mathrm{ml}$, Sigma Chemical Co.l for $\sim 16 \mathrm{hr}$. The mitotic index was assessed by counting mitotic figures in methanol fixed cells whose DNA had been stained fluorescently with bis-benzamide $(0.4 \mu \mathrm{g} / \mathrm{ml}$, Sigma). Synchronized cultures were between $90 \%$ and $95 \% \mathrm{mi}^{-}$ totic at the time of use.

\section{Immunocytochemistry}

Cells grown on cover slips were washed in phosphate-buffered saline (PBS) and then fixed for $10 \mathrm{~min}$ in $4 \%$ paraformaldehyde in PBS. They were then incubated in permeabilization buffer (PBS containing $0.5 \%$ NP-40) for 10 min followed by PBG buffer [PBS containing $0.5 \%$ bovine serum albumin, Fraction 5, Boehringer Mannheim, and $0.1 \%$ gelatin (Sigma)] to block nonspecific antibody binding. Cells were incubated overnight in primary antibody diluted in PBG, washed $4 \times 5$ min with PBG and incubated with lissamine rhodamine (LRSC) conjugated secondary antibodies specific for mouse or rabbit IgG (Jackson ImmunoResearch). Cells were again washed $3 \times 5 \mathrm{~min}$ and DNA was stained by incubation in PBS containing $0.5 \mathrm{ng} / \mathrm{ml}$ DAPI (Sigma) and then mounted in Aquamount (Lerner Laboratories). In Figure 2, cells were extracted with $0.5 \%$ NP-40 in PBS for 2 min before fixation.

\section{Cell fractionation and immunoblotting}

Purified chromosomes used in Figure 2B were prepared according to Gasser and Laemmli (1987). The technique yields pure chromosomes in clusters that allow dilution and counting in a hemocytometer. Cell equivalents were based on the number of chromosome clusters in the final preparation. Protein was extracted by boiling in Laemmli sample buffer (Harlow and Lane 1988) followed by removal of bulk chromosomal DNA by pelleting at $100,000 \mathrm{~g}$ in an ultracentrifuge. Protein from $4 \times 10^{6}$ cells ( 1 cellular equivalent, CE) was loaded onto each lane of a $10 \%$ SDS-polyacrylamide gels for electrophoresis (Harlow and Lane 1988) and subsequent electroblotting (400 mA-2 hr) to PVDF membrane (Immobilon, Millipore). These membranes were blocked by incubation at room temperature for $1 \mathrm{hr}$ with TBSTB buffer $150 \mathrm{~mm}$ Tris at $\mathrm{pH} 7.4,150 \mathrm{~mm} \mathrm{NaCl}, 1 \%$ Tween 20 , and $2 \% \mathrm{BSA}$ ) and then probed for $\sim 2 \mathrm{hr}$ with primary antibody diluted in TBSTB. After washing $4 \times 5^{\prime}$ in TBST (no BSA), primary antibody was detected by incubation with ${ }^{125}$ I-protein A $\left(2-10 \mu \mathrm{Ci} / \mu \mathrm{g}-\sim 0.5 \times 10^{6} \mathrm{DPM} / \mathrm{ml}, \mathrm{NEN}\right)$ diluted in TBSTB for $2-3 \mathrm{hr}$ at $4^{\circ} \mathrm{C}$ and subsequently exposed for autoradiography. When the primary antibody was a mouse monoclonal, the blot was incubated for $1 \mathrm{hr}$ in rabbit antimouse IgG polyclonal antibody diluted in TBSTB for $1 \mathrm{hr}$ before washing and incubation with ${ }^{125}$ I-protein A. In Figure 3, primary antibody was detected by secondary antibody (goat antimouse IgG, Jackson Immunoresearch/ coupled to alkaline phosphatase and reacted according to standard procedures (Harlow and Lane 1988).

\section{Metabolic labeling and immunoprecipitation from lysates}

Metabolic labeling of HeLa cells with ${ }^{32} \mathrm{P}$-orthophosphate was carried out with either log phase cells (mitotic index $<2 \%$ ) or cells synchronized in mitosis by treatment with nocodazole (mitotic index $>93 \%$ ) as described previously (Roberts et al. 1991 ), except that lysis buffer also contained the following phosphatase inhibitors: $60 \mathrm{~mm} \beta$-glycerophosphate, $5 \mathrm{~mm} \mathrm{NaPPi}, 50$ $\mathrm{mM} \mathrm{NaF}, 100 \mu \mathrm{M}$ NaOrthovanadate (Sigma Chemical). TBP was 
immunoprecipitated with polyclonal antibodies raised against the bacterially expressed core domain of TBP but detected on the immunoblot with a monoclonal antibody directed against an epitope in the amino terminus of TBP (gift of Nancy Thompson, McArdle Laboratories). Immunoprecipitates were collected on protein A beads (Zymogen), eluted by boiling in Laemmli sample buffer, separated by SDS-PAGE and transferred to PVDF membrane (Immobilon, Millipore). $\mathrm{hTAF}_{\mathrm{II}}$ 20/15, $\mathrm{hTAF}_{\mathrm{II}} 31$, and $\mathrm{hTAF}_{\mathrm{II}} 80$ were detected with polyclonal antibodies raised in rabbits (Hisatake et al. 1995; Hoffmann et al. 1996). Secondary antimouse and antirabbit IgG were conjugated to alkaline phosphatase (Jackson ImmunoResearch). The immunoblot was exposed for autoradiography before alkaline phosphatase reaction in order to colocalize metabolically incorporated ${ }^{32} \mathrm{p}$-orthophosphate and the alkaline phosphatase reaction product.

\section{Immunoprecipitation for TFIID subunit composition studies}

Whole-cell extracts from log phase and mitotic HeLa cells were made as described previously (Segil et al. 1991) with the addition of inhibitors [60 mM $\beta$-glycerophosphate, $5 \mathrm{~mm} \mathrm{NaPPi}, 50$ $\mathrm{mm} \mathrm{NaF}, 100 \mu \mathrm{M}$ NaOrthovanadate [Sigma Chemical)]. Extracts were not dialyzed but were brought to $20 \%$ glycerol and used directly for immunoprecipitation in BC buffer $120 \mathrm{~mm}$ HEPES of $\mathrm{pH} 7.9,20 \%$ glycerol, $0.5 \mathrm{~mm}$ dithiothreitol, $0.5 \mathrm{mM}$ PMSF) with $400 \mathrm{~mm} \mathrm{KCl}$. Immunoprecipitates were collected on protein A beads (Zymogen) and eluted by boiling in Laemmli sample buffer. Proteins were separated by SDS-PAGE and transferred and probed as described above.

\section{Preparation of purified $f:$ TFIID and in vitro transcription}

Whole-cell extracts (Segil et al. 1991) were prepared from HeLa cells expressing FLAG-tagged TBP (Chiang et al. 1993) and synchronized in mitosis by nocodazole block. Extracts were prepared and dialyzed in $\mathrm{BC} 100$ buffer in either the presence or absence of the same cocktail of phosphatase inhibitors used for the preparation of lysates in the metabolic-labeling experiments. TFIID was immunoprecipitated on anti-FLAG $\mathrm{M}_{2}$ agarose (Kodak/IBI) as described previously (Chiang et al. 1993) except that immunoprecipitation took place directly from whole-cell extracts with no prior fractionation. The reconstituted in vitro transcription system used to test the purified TFIID was prepared and transcription reactions carried out as described previously (Chiang et al. 1993). Two plasmids containing G-less cassettes of different lengths were used to analyze basal and activator-dependent transcription simultaneously. Basal transcription was assayed with plasmid pML $\Delta 53$, which contains the adenovirus major late promoter TATA box and transcription initiation sequence and activator-dependent transcription was assayed with $\mathrm{pG}_{5} \mathrm{HMC}_{2} \mathrm{AT}$, which contains the HIV TATA sequence and adenovirus major late promoter (MLP) transcription initiation sequences cloned downstream from five tandem copies of the Gal4 DNA-binding motif (Sawadogo and Roeder 1985; Chiang et al. 1993). Bacterially expressed FLAGGal4 fusion proteins were purified as described previously (Lin et al. 1988; Chiang et al. 1993) and consist of amino-acids 1-96 of the Gal4 DNA-binding domain fused to a duplicated copy of the transcriptional activation domain from p 53 (amino acids 1-57) or a single copy of the SPI transcriptional activation domain (amino acids 82-500) (M. Guermah, unpubl.).

\section{Acknowledgments}

We thank Wendy Lee for expert tissue culture support, Don Arnold, Stephen Burley, Cheng-Ming Chiang, Andrew Czernik,
Lisa Dailey, Titia deLange, Eva Dworkin, Susan Roberts, Greta Segil, and Bas van Steensel for advice, support, and/or careful reading of the manuscript, and Dr. Nancy Thompson and Dr. Nouria Hernandez for the anti-TBP monoclonal antibodies used in this study. "This study was made possible, in part, by a fund granted to N.S. through a fellowship program sponsored by the Charles Revson Foundation. The statements made and views expressed are solely the responsibility of the author." A.H. was a recipient of a fellowship from Boehringer Ingelheim Fonds. This work was supported by U.S. Public Health Service Grant GM-32544 from the National Institutes of Health (NIH) and The Howard Hughes Medical Institute (N.H.) and U.S. Public Health Service grant no. CA42567 from the NIH (R.G.R.).

The publication costs of this article were defrayed in part by payment of page charges. This article must therefore be hereby marked "advertisement" in accordance with 18 USC section 1734 solely to indicate this fact.

\section{References}

Brown, D.D. 1984. The role of stable complexes that repress and activate eukaryotic genes. Phil. Trans. R. Soc. Lond. B. Biol. Sci. 307: 297-299.

Burley, S.K. and R.G. Roeder. 1996. Biochemistry and structural biology of transcription factor IID (TFIID). Annu. Rev. Biochem. 65: 769-799.

Chen, J.-L., L.D. Attardi, C.P. Verrijzer, K. Yokomori, and R. Tjian. 1994. Assembly of recombinant TFIID reveals differential coactivator requirements for distinct transcriptional activators. Cell 79: 93-105.

Chiang, C.-M., H. Ge, Z. Wang, A. Hoffmann, and R.G. Roeder. 1993. Unique TATA-binding protein-containing complexes and cofactors involved in transcription by RNA polymerases II and III. EMBO I. 12: 2749-2762.

Davidson, D. 1964. RNA synthesis in roots of Vicia faba. Exp. Cell Res. 35: 317-325.

Delong, I. and R.G. Roeder. 1993. A single cDNA, hTFIIA/ $\alpha$, encodes both the p35 and p19 subunits of human TFIIA. Genes \& Dev. 7: 2220-2234.

Dynlacht, B.D., T. Hoey, and R. Tjian. 1991. Isolation of coactivators associated with the TATA-binding protein that mediate transcriptional activation. Cell 66: 563-576.

Farber, J., G. Stein, and R. Baserga. 1972. The regulation of RNA synthesis during mitosis. Biochem. Biophys. Res. Comm. 47: 790-797.

Gasser, S.M. and U.K. Laemmli. 1987. Improved methods for the isolation of individual and clustered mitotic chromosomes. Exp. Cell Res. 173: 85-98.

Gauthier-Rouviere, C., I.-C. Cavadore, J.-M. Blanchard, N.J.C. Lamb, and A. Fernande. 1991. p67SRF is a constitutive nuclear protein implicated in the modulation of genes required throughout the Gl period. Cell Reg. 2: 575-588.

Goodrich, J.A., G. Cutler, and R. Tjian. 1996. Contacts in context: Promoter specificity and macromolecular interactions in transcription. Cell 84: 825-830.

Gottesfeld, J.M., V.J. Wolf, T. Dang, D.J. Forbes, and P. Hartl. 1994. Mitotic repression of RNA polymerase III transcription in vitro mediated by phosphorylation of a TFIIIB component. Science 263: 81-84.

Greenberg, M.E. and E.B. Ziff. 1984. Stimulation of 3T3 cells induces transcription of the c-fos proto-oncogene. Nature 311: 433-438.

Harlow, E. and D. Lane. 1988. Antibodies: A laboratory man- 
ual. Cold Spring Harbor Laboratory, Cold Spring Harbor, NY.

Hartl, P., J. Gottesfeld, and D. J. Forbes. 1993. Mitotic repression of transcription in vitro. J. Cell Biol. 120: 613-624.

Hershkovitz, M. and A.D. Riggs. 1995. Metaphase chromosome analysis by ligation-mediated PCR: Heritable chromatin structure and a comparison of active and inactive $\mathrm{X}$ chromosomes. Proc. Natl. Acad. Sci. 92: 2379-2383.

Hisatake, K., T. Ohta, R. Takada, M. Guermah, M. Horikoshi, Y. Nakatani, and R.G. Roeder. 1995. Evolutionary conservation of human TATA-binding-polypeptide-associated factors TAF31 and TAF80 and interactions of TAF80 with other TAFs and with general transcription factors. Proc. Natl. Acad. Sci. 92: 8195-8199.

Hoffmann, A. and R.G. Roeder. 1996. Cloning and characterization of human TAF20/15: Multiple interactions suggest a central role in TFIID complex formation. J. Biol. Chem. 271: 18194-18202.

Hoffmann, A., C.-M. Chiang, T. Oelgeschläger, X. Xie, S.K. Burley, Y. Nakatani, and R.G. Roeder. 1996. A histone octamerlike structure within TFIID. Nature 380: 356-359.

Hori, R. and M. Carey. 1994. The role of activators in assembly of RNA polymerase II transcription. Curr. Opin. Gen. Dev. 4: $236-244$.

Horikoshi, M., M.F. Carey, H. Kakidani, and R.G. Roeder. 1988a. Mechanism of action of a yeast activator: Direct effect of GAL4 derivatives on mammalian TFIID-promoter interactions. Cell 54: 665-669.

Horikoshi, M., T. Hai, Y.S. Lin, M.R. Green, and R.G. Roeder. 1988b. Transcription factor ATF interacts with the TATA factor to facilitate establishment of a preinitiation complex. Cell 54: 1033-1042.

Hsu, S.C., M. Qi, and D.B. DeFranco. 1992. Cell cycle regulation of glucocorticoid receptor function. EMBO /. 11: 3457-3468.

Johnson, T.C. and J.J. Holland. 1965. Ribonucleic acid and protein synthesis in mitotic HeLa cells. I. Cell Biol. 27: 565574.

Jordan, P., M. Mannervik, L. Tora, and M. Carmo-Fonesca. 1996. In vivo evidence that TATA-binding protein/SL1 colocalizes with UBF and RNA polymerase I when rRNA synthesis is either active or inactive. I. Cell Biol. 122: 225-234.

Kerem, B.S., R.Goitein, G. Diamond, H. Cedar, and M. Marcus. 1984. Mapping of DNase I sensitive regions on mitotic chromosomes. Cell 38: 493-499.

Kokubo, T., D.-W. Gong, J.C. Wooton, M. Horikoshi, and R.G. Roeder. 1994. Molecular cloning of Drosophila TFIID subunits. Nature 367: 484-487.

Laurenson, P. and J. Rine. 1992. Silencers, silencing, and heritable transcriptional states. Microbiol. Rev. 56: 543-560.

Lieberman, P.M. and A.J. Berk. 1994. A mechanism for TAFs in transcriptional activation: Activation domain enhancement of TFIID-TFIIA promoter DNA complex formation. Gen. \& Dev. 8: 995-1006.

Lin, Y.S., M.F. Carey, M. Ptashne, and M.R. Green. 1988. GAL4 derivatives function alone and synergistically with mammalian activators in vitro. Cell 54: 659-664.

Lobo, S.M., M. Tanaka, M.L. Sullivan, and N. Hernandez. 1992. A TBP complex essential for transcription from TATA-less but not TATA-containing RNA polymerase III promoters is part of the TFIIIB fraction. Cell 71: 1029-1040.

Lu, H. and A.J. Levine. 1995. Human Taf31 protein is a transcriptional coactivator of the p53 protein. Proc. Natl. Acad. Sci. 92: 5145-5148.

Luscher, B. and R.N. Eisenman. 1992. Mitosis-specific phosphorylation of the nuclear oncoproteins Myc and Myb. J. Cell Biol. 118: 775-784.
Malik, S., D.K. Lee, and R.G. Roeder. 1993. Potential RNA polymerase II-induced interactions of transcription factor TFIIB. Mol. Cell. Biol. 13: 6253-6259

Martinez-Balbas, M., A. Dey, S.K. Rabindran, K. Ozata, and C. Wu. 1995. Displacement of sequence-specific transcription factors from mitotic chromatin. Cell 83: 29-38.

Matsui, S.-I., H. Weinfeld, and A.A. Sandberg. 1979. Quantitative conservation of chromatin-bound RNA polymerases I and II mitosis. Implication for chromosome structure. J. Cell Biol. 80: 451-464.

Mengus, G., M. May, X. Jacq, A. Staub, L. Tora, P. Chambon, and I. Davidson. 1995. Cloning and characterization of hTAFII18, hTAFII20 and hTAFII28: Three subunits of the human transcription factor TFIID. EMBO J. 14: 1520-1531.

Mitchell, P.J. and R. Tjian. 1989. Transcriptional regulation in mammalian cells by sequence-specific DNA binding proteins. Science 245: 371-378.

Nurse, P. 1990. Universal control mechanism regulating onset of M-phase. Nature 344: 503-508.

Prescott, D.M. 1976. The cell cycle and the control of cellular reproduction. In Advances in genetics (ed. E.W. Caspari), pp. 99-177. Academic Press, New York, NY.

Prescott, D.M. and M.A. Bender. 1962. Synthesis of RNA and protein during mitosis in mammalian tissue culture cells. Exp. Cell Res. 26: 260-268.

Raff, J.W., R. Kellum, and B. Alberts. 1994. The Drosophila GAGA transcription factor is associated with specific regions of heterochromatin throughout the cell cycle. $E M B O F$. 13: $5977-5983$.

Roberts, S.B., N. Segil, and N. Heintz. 1991. Differential phosphorylation of the transcription factor Octl during the cell cycle. Science 253: 1022-1026.

Roeder, R.G. 1996. The role of general initiation factors in transcription by RNA polymerase II. Trends Biochem. Sci. 21: 327-335.

Roussel, P., C. Andre, G. Masson, G. Geraud, and D. HernandezVerdun. 1993. Localization of the RNA polymerase I transcription factor hUBF during the cell-cycle. I. Cell Sci. 104: 327-337.

Roussel, P., C. Andre, L. Comai, and D. Hernandez-Verdun. 1996. The rDNA transcription machinery is assembled during mitosis in active NOR's and absent in inactive NORs. J. Cell Biol. 133: 235-246.

Sahasrabuddhe, C.G., R.C. Adlakha, and P.N. Rao. 1984. Phosphorylation of non-histone proteins associated with mitosis in HeLa cells. Exp. Cell Res. 153: 439-450.

Sawadogo, M. and R.G. Roeder. 1985. Factors involved in specific transcription by human RNA polymerase II: Analysis by a rapid and quantitative in vitro assay. Proc. Natl. Acad. Sci. 82: 4394-4398.

Scheer, U. and K.M. Rose. 1984. Localization of RNA polymerase I in interphase cells and mitotic chromosomes by light and electron microscopic immunocytochemistry. Proc. Natl. Acad. Sci. 81: 1431-1435.

Segil, N., S.B. Roberts, and N. Heintz. 1991. Mitotic phosphorylation of the Oct-1 homeodomain and regulation of Oct-1 DNA binding activity. Science 254: 1814-1816.

Shermoen, A.W. and P.H. O'Farrell. 1991. Progression of the cell cycle through mitosis leads to abortion of nascent transcripts. Cell 67: 303-310.

Simmons, T., P. Heywood, and L. Hodge. 1973. Nuclear envelope-associated resumption of RNA synthesis in late mitosis of HeLa cells. I. Cell Biol. 59: 150-164.

Taylor, J. 1960. Nucleic acid synthesis in relation to the cell division cycle. Ann. N.Y. Acad. Sci. 90: 409-421.

Terasima, T. and L.J. Tolmach. 1963. Growth and nucleic acid 
Segil et al.

synthesis in synchronously dividing populations of $\mathrm{HeLa}$ cells. Exp. Cell Res. 30: 344-362.

Thut, C.J., J-L. Chen, R. Klemm, and R. Tjian. 1995. p53 transcriptional activation mediated by coactivators $\mathrm{TAF}_{11} 40$ and $\mathrm{TAF}_{\mathrm{II}}$ 60. Science 267: 100-104.

Tjian, R. and T. Maniatis. 1994. Transcriptional activation: A complex puzzle with few easy pieces. Cell 77: 5-8.

Villarreal, L.P. 1991. Relationship of eukaryotic DNA replication to committed gene expression: General theory for gene control. Microbiol. Rev. 55: 512-542.

Weintraub, H. 1985. Assembly and propagation of repressed and depressed chromosomal states. Cell 42: 705-711.

Weisenberger, D. and U. Scheer. 1995. A possible mechanism for the inhibition of ribosomal RNA gene transcription during mitosis. J. Cell Biol. 129: 561-575.

White, R.J., T.M. Gottlieb, C.S. Downes, and S.P. Jackson. 1995. Mitotic regulation of a TATA-binding-protein-containing complex. Mol. Cell. Biol. 15: 1983-1992.

Wolffe, A.P. 1994. Inheritance of chromatin states. Dev. Genet. 15: $463-470$.

Workman, J.L., S.M. Abmayr, W.A. Cromlish, and R.G. Roeder. 1988. Transcriptional regulation by the immediate early protein of pseudorabies virus during in vitro nucleosome assembly. Cell 55: 211-219.

Xie, X., T. Kokubo, S. L. Cohen, U.A. Mirza, A. Hoffmann, B.T. Chait, R.G. Roeder, Y. Nakatani, and S.K. Burley. 1996. Structural similarity between TAFs and the heterotetrameric core of the histone octamer. Nature 380: 316-322.

Zawel, L. and D. Reinberg. 1993. Initiation of transcription by RNA polymerase II: A multi-step process. Prog. Nucleic Acid Res. Mol. Biol. 44: 67-108.

- 1995. Common themes in assembly and function of eukaryotic transcription complexes. Annu. Rev. Biochem. 64: 533-561.

Zhou, Q., P.M. Lieberman, T.G. Boyer, and A.J. Berk. 1992. Holo-TFIID supports transcriptional stimulation by diverse activators and from a TATA-less promoter. Genes \& Dev. 6: 1964-1974.

Zhou, Q., T.G. Boyer, and A.J. Berk. 1993. Factors (TAFs) required for activated transcription interact with TATA boxbinding protein conserved core domain. Genes \& Dev. 7: 180-187. 


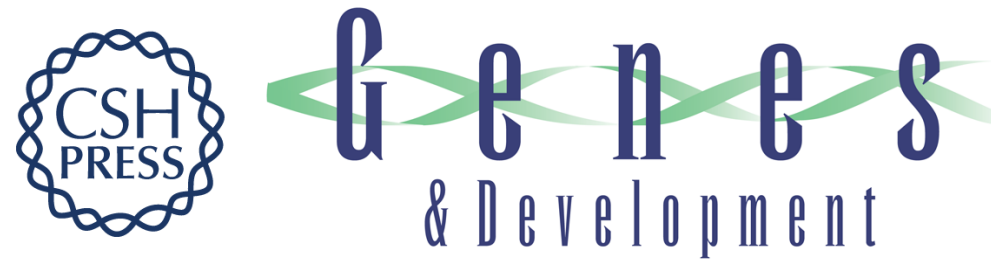

\section{Mitotic regulation of TFIID: inhibition of activator-dependent transcription and changes in subcellular localization.}

N Segil, M Guermah, A Hoffmann, et al.

Genes Dev. 1996, 10:

Access the most recent version at doi:10.1101/gad.10.19.2389

References This article cites 65 articles, 26 of which can be accessed free at:

http://genesdev.cshlp.org/content/10/19/2389.full.html\#ref-list-1

License

Email Alerting

Service

Receive free email alerts when new articles cite this article - sign up in the box at the top right corner of the article or click here.

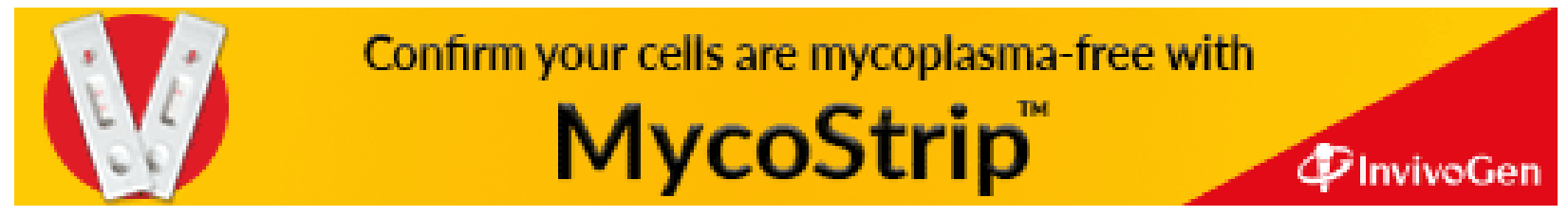

